\title{
Transforming response measures to remove interactions or other sources of variance ${ }^{1}$
}

RICHARD S. BOGARTZ and JOHN $H$. WACKWITZ, University of Illinois, Urbana, Ill. 61801

A general polynomial transformation is given for the dependent variable in experimental design models and multiple linear or multiple polynomial regression models such that selected sources of variance such as interactions or configuralities are reduced or eliminated. $A$ stopping rule is given for addition of terms in the polynomial based on proportion of systematic (nonerror) variance accounted for by the selected source.

Statistically significant interactions are not always psychologically meaningful. Often they merely reflect the particular choice of a measurement scale for the response. Sometimes, general familiarity with the occurrence of such interactions is sufficient to avoid problems. Other times, ignorance of or inadequate handling of the response scale problem can have ramifications throughout an entire area of investigation. Interactions that may well be attributable to inadequacies of the response measurement scale are often routinely taken as revealing psychological processes or mechanisms.

Ceiling effects and floor effects are familiar instances in which the presence of a significant interaction is discounted. Figure 1 shows two error probability functions, one for low drive and the other for high drive. The bigger difference between the curves at Trial 8 than at Trial 24 would produce a statistical interaction. It would be discounted as due to a floor effect rather than attributed to some meaningful interaction of drive level with trials.

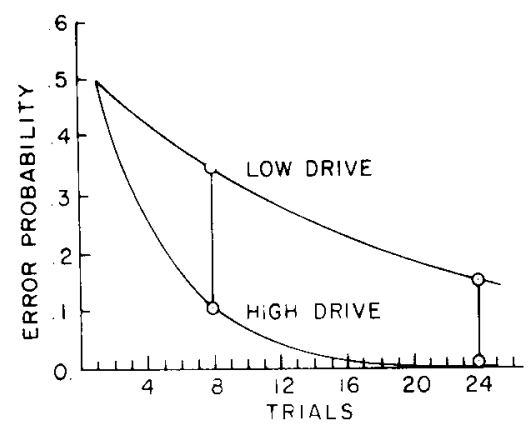

Fig. 1. Graphical representation of an interaction due to a floor effect.
To see the role of the response scale in determining the presence or absence of an interaction, assume that the functions in Fig. 1 are $.5 \mathrm{a}^{\mathrm{n}-1}$ and $.5 b^{n-1}$, where $n$ is the trial number. Treating trial number and drive levels as the two factors of a 2 by 2 factorial design, we can see that the interaction effect comparison is then $\left(.5 \mathrm{a}^{8-1}-\right.$ $\left..5 \mathrm{~b}^{8-1}\right)-\left(.5 \mathrm{a}^{24-1}-.5 \mathrm{~b}^{24-1}\right)$, and will be nonzero if $a \neq b$. However, if instead of error probability, the measure is $\log \log$ [2(error probability)l, the interaction comparison becomes $\left[\log \log 2\left(.5 a^{7}\right)-\log \log 2\left(.5 b^{7}\right)\right]-$ $\left\{\log \log \left[2\left(.5 \mathbf{a}^{\mathbf{2 3}}\right)\right]-\log \log \left[2\left(.5 b^{\mathbf{2}}\right)\right\}^{\prime}\right.$, which is zero for all $a$ and $b$. The intuitive notion of $a$ floor effect as the basis for the interaction is here made rigorous by demonstrating a response measure transformation that scales out the interaction. Since the presence or absence of the interaction depends only on the choice of the measurement scale, it is psychologically meaningless in the sense that it indicates no psychological process.

When numerical rating scales are used, distortions produced by, say, end effects or number preferences can also introduce psychologically meaningless interactions. Figure 2 shows an example of end effects in which bigger differences in the psychological variable $V$ are required at the scale ends than at the scale middle to produce a given difference in the numerical rating response, $R$. If an $A$ by $B$ factorial design with two levels of $A$ and three levels of $B$ were used to study the relation of the psychological variable $V$ to the two independent variables $A$ and $B$, means of 11,13 , and 15 for the three levels of $B$ at the first level of $A$, and of 13,15 , and 17 for the three levels of $B$ at the second level of $A$ would show an additive relation. The function shown in Fig. 2 indicates that such additivity at the level of the psychological variable $V$ would be misrepresented as interaction by the numerical response $R$. On the numerical response scale, the values 11,13 , and 23 would be obtained at the first level of $A$ and the values 13,23 , and 29 at the second level of $\mathrm{A}$. The apparent interaction does not reflect a nonadditive relation of $A$ and $B$ to the psychological variable. Rather, it reveals the end effects characterizing the Ss' use of the numerical rating response.

Just as in the first example, where a transformation of the response measure scaled out the interaction, so too, in this second example, such a transformation of the numerical rating response measure is possible. Since the function $f$ relating $R$ to $\mathrm{V}$ is strictly monotonic, the inverse function $f^{-1}$ exists such that $V=f^{-1}(R)$. This constitutes a rescaling of the dependent variable to a new psychologically correct response measure in which the interaction has been removed.

As a third example, consider a theory in which drive level (D) and incentive level (I) are assumed to combine additively to yield an intervening variable, $E$, which determines response speed according to the law, $S=E^{2}$. If a factorial design is used to manipulate $D$ and $I$ orthogonally, additivity of drive and incentive will be concealed by the nonlinear relation of speed to $\mathrm{E}$. Thus Table 1 gives an additive relation for the cell values of $D+I=E$, but the speed scores, equal to $\mathrm{E}^{2}$, will be those shown in Table 2. The apparent interaction in the table of speed scores would be removed and the underlying additivity would be revealed by a square root transformation of the speed scores.

Note that in both the second and third example, rescaling of the dependent variable to eliminate interaction was achieved using information that is ordinarily not available, namely the functional relation of the dependent variable to the underlying variable.

Clearly, for situations such as these, it would be desirable to have a statistical technique for rescaling the dependent variable that would remove interactions even when the functional relation of the dependent variable to the underlying variable is not known. Ideally, the technique would yield a simple, explicit, usually monotonic transformation of the dependent variable to a new scale on which those interactions due only to the choice of scale would be zero.

We report here, in preliminary, abbreviated form, a new statistical method for transforming the dependent variable in an analysis of variance (ANOVA), such that the variance due to any selected source or sources is reduced or removed. The transformation is of the form: $S^{*}=S+a_{2} S^{2}+\cdots+a_{n} S^{n}$, whereby $S$, the original score, is transformed to $S^{*}$. The method selects the $a_{i}$ to minimize the

Table 1

Drive and Incentive Levels

\begin{tabular}{|c|c|c|c|c|c|}
\hline & & \multicolumn{4}{|c|}{ I } \\
\hline & & 1 & 2 & 3 & 4 \\
\hline \multirow{3}{*}{ D } & 1 & 2 & 3 & 4 & 5 \\
\hline & 2 & 3 & 4 & 5 & 6 \\
\hline & 3 & 4 & 5 & 6 & 7 \\
\hline
\end{tabular}

Table 2

Speed Scores $\left(\mathbf{E}^{2}\right)$

\begin{tabular}{|c|c|c|c|c|c|}
\hline & & \multicolumn{4}{|c|}{ I } \\
\hline & & 1 & 2 & 3 & 4 \\
\hline $\mathrm{D}$ & $\begin{array}{l}1 \\
2 \\
3\end{array}$ & $\begin{array}{r}4 \\
9 \\
16\end{array}$ & $\begin{array}{r}9 \\
16 \\
25\end{array}$ & $\begin{array}{l}16 \\
25 \\
36\end{array}$ & $\begin{array}{l}25 \\
36 \\
49\end{array}$ \\
\hline
\end{tabular}


selected source(s) and provides a stopping rule for how many $a_{j}$ terms are needed. The method is appropriate to all instances of the General Linear Model (Mood \& Graybill, 1963), including fixed-effects experimental design models, multiple linear, and multiple polynomial regression models.

Our original concern was finding a transformation to additivity, i.e., removing interactions. We have noted, however, that our method is also appropriate to removing configuralities in regression models for simulated clinical judgment (Wiggins \& Hoffman, 1968) and to removing orthogonal trend components, e.g., cubic, quartic, linear by cubic, from the main effects of interactions in experimental-design models. Although these last applications, such as removing the linear by cubic component by a scale transformation, appear at first glance to be remote from any practical psychological problems, they turn out to have a rather wide range of important applications that we shall describe in future, more extensive reports on this method.

\section{RESULTS}

After the source to be removed has been found to be significant, the polynomial transformation is used, beginning with the quadratic transformation $\mathrm{S}^{*}=\mathrm{S}+\mathrm{a}_{2} \mathrm{~S}^{2}$ and incrementing the polynomial by one term until stopping. The decision as to whether to stop or to add another term to the polynomial is made as follows. A new ANOVA is done after each new term is added to the polynomial. One $\mathrm{df}$ is subtracted from the source being removed for each $a_{i}$ used. The df are then only approximately correct. In each ANOVA, the proportion of systematic (nonerror) variance accounted for by the reduced source can be estimated. Addition of terms to the polynomial can stop when this proportion is less than .05 or when the $\mathrm{df}$ have been used up.

For any instance of the General Linear Model in which we seek to remove a source of variance, we express a larger hypothesis, $\Omega$, which includes that source, and a smaller hypothesis, $\omega$, in which it has been removed. Figure 3 shows this in vector-space form for the case of removing interactions in an experimental design model. After transformation, the model will be $S^{*}=X^{\prime} \Omega \beta \Omega+e \Omega$ under $\Omega$ and $S^{*}=X^{\prime}{ }_{\omega}{ }{ }_{\omega}+e_{\omega}$ under $\omega$, where in each case $\beta$ is a vector of unknown parameters, $\mathrm{X}^{\prime}$ is a matrix of measured qualitative or quantitative weights, and $e$ is a vector of errors.

The error sum of squares for the two models will be of the form $\mathrm{e}_{\mathrm{a}_{\mathrm{a}}} \mathrm{e}_{\mathrm{a}}=$ $\left(S^{*}-X^{\prime} \beta_{a}\right)^{\prime}\left(S^{*}-X^{\prime}{ }_{a} \beta_{a}\right), a=\Omega, \omega$. The sum of squares to be reduced will be their

Fig. 3. A vector-space representation of the various vectors of interest and their squared lengths, the various sums of squares of interest.

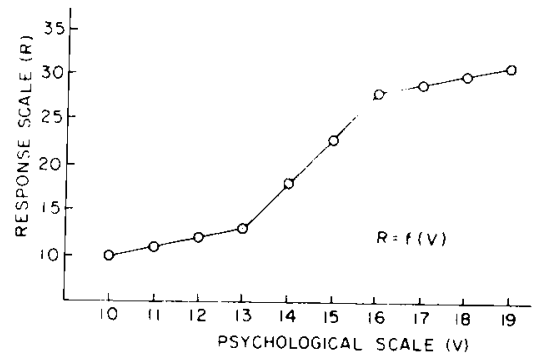

Fig. 2. Hypothetical functional relationship $f$ between an underlying psychological variable $\mathrm{V}$ and an overt rating response $R$, showing end effects in the use of a rating scale.

difference, $\quad \Sigma=\mathrm{e}^{\prime} \omega^{\mathrm{e}} \omega-\mathrm{e}^{\prime} \Omega \mathrm{e} \Omega=$ $S^{* \prime}\left(R_{\Omega}-R_{\omega}\right) S^{*}$, where $R_{\Omega}=X^{\prime} \Omega$ $\left(\mathrm{X}_{\Omega} \mathrm{X}^{\prime} \Omega\right)^{-1} \mathrm{X}_{\Omega}$ and $\mathrm{R}_{\omega}=\mathrm{X}_{\omega}^{\prime}$ $\left(\mathrm{X}_{\omega} \mathrm{X}_{\omega}^{\prime}\right)^{-1} \mathrm{X}_{\omega}$.

Since $\Sigma$ depends on $S^{*}$, we seek those a which will minimize $\Sigma$. Let $a_{n}$ be the vector $\left(\begin{array}{lllll}1 & a_{2} & a_{3} & \cdots & a_{n}\end{array}\right)$ and $S_{n}$ be the matrix $\left(S S^{2} S^{3} \cdots S^{n}\right)$, where the $i$ th element in $S^{j}$ is the $j^{\text {th }}$ power of the $i^{\text {th }}$ element in $S$. Then $S^{*}{ }_{n}=S_{n} a_{n}$, so that $\Sigma=a_{n} S_{n}^{\prime}\left(R \Omega-R_{\omega}\right) S_{n} a_{n}^{\prime}=a_{n} D_{n} a^{\prime}$. It is then easy to show that if the $n \times n$ matrix $D_{n}$ is partitioned into the $1 \times 1,1 \times n-1$, $\mathrm{n}-1 \times 1$, and $\mathrm{n}-1 \times \mathrm{n}-1$ submatrices $d_{11}, D_{12}, D_{21}$, and $D_{22}$, and if we write $a_{n}=\left[1 \mid a_{2} a_{3} \cdots a_{n}\right]=\left[1 \mid A_{n}\right]$, then the vector of $a_{i}$ 's $(i=2-n)$, which minimizes $\Sigma$, is just $A_{n}=(-1)\left(D_{22}{ }^{-1} D_{21}\right)^{\prime}$.

With the standard computer routines for matrix multiplication and inversion that are widely available, a computer program to perform the computations for this problem is a simple matter. A program in Fortran IV is available upon request.

\section{NUMERICAL EXAMPLES}

Figure 4 shows the removal of an interaction suggestive of a floor effect. The $F(4,18)$ for interaction in the original data $\left(\mathrm{T}_{0}\right)$ is 19.84 . Transforming to $S^{*}=S-.0065038 \mathrm{~S}^{2}$ gives the means plotted as Transformation $T_{1}$ and an $F(3,18)=3.14$. Transformation $T_{2}$ : $\mathrm{S}^{*}=\mathrm{S}-.014567 \mathrm{~S}^{2}+.000081213 \mathrm{~S}^{3}$ gave the third plot of means and an $F(2,18)=.50$.

For our second numerical example, we return to the endeffects problem discussed in the second example of the introduction. A 3 by 5 factorial design of cell means, showing no in teraction when measured on the psychological scale $V$, was constructed. These values were $11,12,13,14,15: 13$, $14,15,16,17$; and $15,16,17,18,19$. Using the function in Fig. 2, these cell means were transformed to cell means of the response measure. giving means of $11,12,13,18,23 ; 13,18,23,28$, 29 ; and $23,28,29,30,31$. Variability was then built in about these means.

In Fig. 5, Panel a shows the mean $R$ values for the 15 cells. The $F(8,135)$ for interaction is 14.40 . Panel $b$ shows the means for the transformed scores $\mathrm{S}^{*}=\mathrm{R}-.04875 \mathrm{R}^{2}+.0007873 \mathrm{R}^{3}$ after they have reccived a linear transformation putting them on the same scale as the $R$ values. The $F(6,135)$ for interaction is 33 . Panel c shows the $S^{*}$ values after application of a linear transformation to put them on the same scalc as the original $V$ values. The lines represent the $V$ values, and the points are the $S^{*}$ values. The interaction in the $R$ values has been scaled out and the scores have been transformed to a linear transformation of the psychologically correct V scale.

\section{DISCUSSION}

The method should be very useful in applications of functional measurement theory (Anderson, in press). In functional measurement, the development of a substantive theory of behavior and the development of measurement scales for the psychological variables in that theory are integrally related. Validation of a measurement scale depends upon establishment of the empirical validity of the theory. By the use of factorial designs (see below) together with the establishment of a correspondence between the customary terms in an experimental-design model and the terms of the substantive theory under investigation, the ANOVA often plays an important role both in testing the correctness of the theoretical structure and in obtaining measurement scales for both stimuli and responses (Anderson, 1962a, b, 1968, 1969; Anderson \& Jacobson, 1968).

The use of numerical response measures in functional measurement makes scaling of the response measures crucial to evaluation of the theory. The correspondence of experimental-design model terms to theoretical terms permits, say, the prediction of zero interactions (Anderson, 1962a). Such a prediction, however, could easily be apparently disconfirmed due to the influence of the measurement scale on the presence or

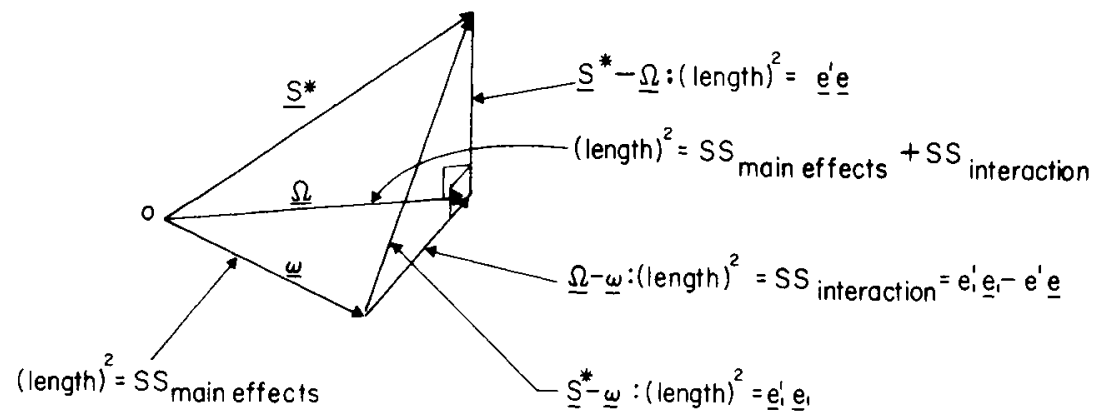


Fig. 4. Graphical presentation of group means showing reduction of interaction from original data $\left(T_{0}\right)$ through two successive transformations $\left(T_{1}, T_{2}\right)$, for Example 1.

absence of psychologically meaningless interactions. Obtaining a monotone transformation of the response measure in such a case not only can save a correct theory, but has the additional virtue of providing a valid response scale. In other contexts, such as a multiplicative model for psychophysical judgment (Anderson, in press), an appropriate transformation will remove some components of the obtained interaction but leave others that are predicted, such as the linear by linear component of a two-way interaction (see below). The method presented here, because of its great versatility in being able to focus upon reduction of variance due to any selected source or set of sources, is therefore ideally suited to such applications.

A word of caution. A powerful tool for scaling out interactions or other sources of variance can be abused. Functional measurement places such scaling into a theoretical context, providing a rationale for the scaling and a test of its validity. In the absence of such a context, response scale decisions can be difficult and will require care.

In this regard, psychological considerations are more important than mathematical considerations. Anderson (in press) cites an in teresting treat ment of a word-photograph interaction found by Lampel \& Anderson (1968). They decided not to rescalc the response to remove the interaction. Their decision was based on confidence in the response scale generated by a body of previous experimentation. Instead of transforming to additivity, they gave a psychological in terpretation to the interaction. Thus, while on abstract mathe matical grounds additivity might have been considered, on psychological grounds it was rejected.

Another example where overtransforming can be a tactical error is the case where the presence or absence of the interaction constitutes the empirical basis for deciding between two models. Anderson (in press) presents an example of this in the analysis of an averaging and a multiplicative model for multisection judgments (a generalization of the classical bisection task). A theoretica analy sis was applied to a three-way factorial design in which the rows and columns of the design represent physical reference stimuli defining the endpoints of an interval, and the third factor is section ratios. The interval is to be divided by the $S$ into intervals that are in the given ratio. Theoretical analysis revealed that after a response transformation to remove the row-column interaction disappearance of interactions of the sections factor with rows and columns would support the multiplicative model, but according to the averaging model these interactions should be present and concentrated in the linear by linear component. In this context, scaling out the linear by linear com-

Fig. 5. A rescaling of data from the $R$ scale back to the $V$ scale, removing the interaction introduced by the end effects.

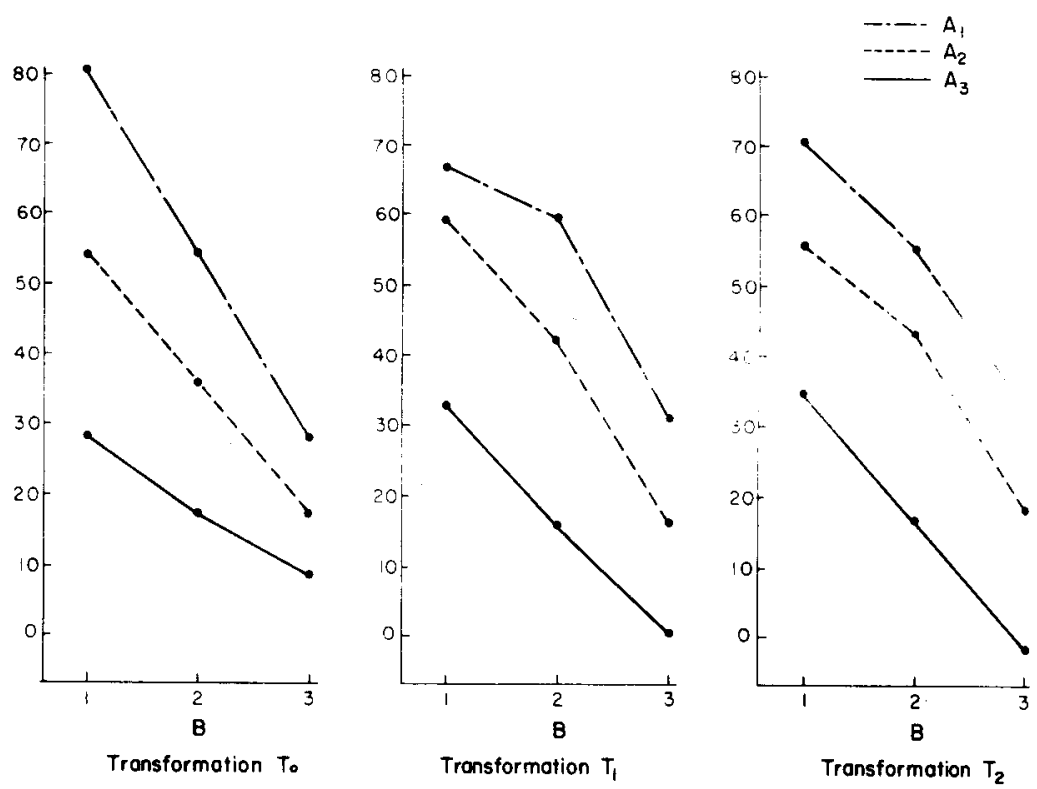

ponent would disable the experiment as a test between the two models.

We should mention that the method presented here does not force a monotone transfornation. In functional measurement and certain other approaches. monotonicity may be required. When this is the case, if the obtained transformation is monotonic, no problem arises. If it is not, constraints on the $a_{i}$ can be introduced which force monotonicity.

REFERENCES

ANDERSON, N. H. Application of an additive model to impression formation. Science 1962a, 138, 817-818.

ANDERSON, N. H. On the quantification of Miller's conflict theory. Psychological Review, $1962 b, 69,400-414$.

ANDERSON, N. H. A simple model for information integration. In R. P. Abelson, $E$ Aronson, W. J. McGuire, T. M. Newcomb, M. J. Rosenberg, and P. H. Tannenbaum (Eds.), Theories of cognitive consistency: $A$ sourcebook. Chicago: Rand McNally, 1968.

ANDERSON, N. H. Comment on "An analysis-of-variance model for the assessment of configural cue utilization in clinical judgment. Psychological Bulletin, 1969, 72. 63-65.

ANDERSON, N. H. Functional measurement and psychophysical judgment. Psychological Review, in press.

ANDERSON, N. H., \& JACOBSON, A. Further data on a weighted average model for judgment in a lifted weight task. Perception \& Psychophysics, 1968, 4, 81-84.

LAMPEL, A., \& ANDERSON, N. H. Combining visual and verbal information in an impression-formation task. Journal of Personality \& Social Psychology, 1968, 9, 1-6. MOOD, A. M., \& GRAYBILL, F. A. Introduction to the theory of statistics (2nded.) New York: McGraw-Hill, 1963.

WIGGINS, N., \& HOFFMAN, P. J. Three models of clinical judgment. Joumal of Abnormal Psychology, 1968, 73, 70-77.

NOTE

1. We are indebted to Norman $H$. Anderson who suggested an investigation of this problem. Portions of this work were supported by PHS Research Grant No. HD-03574-01.
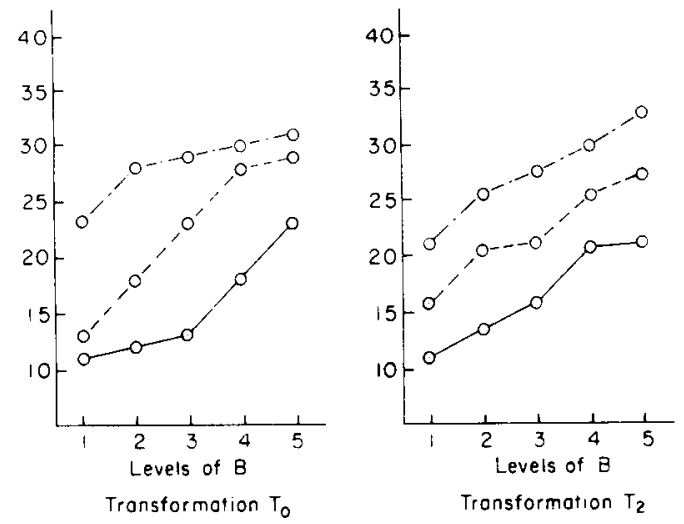

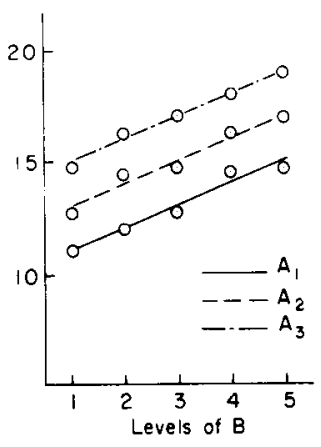

Original V Scores (lines) and Transformation $T_{2}$ (points) ofter linear tronsform 